\title{
ON THE MULTIPLICITY OF THE ZERO-AGE MAIN-SEQUENCE O STAR HERSCHEL 36
}

\author{
Julia I. Arias ${ }^{1}$, Rodolfo H. Barba ${ }^{1,2}$, Roberto C. Gamen ${ }^{3}$, Nidia I. Morrell ${ }^{4}$, Jestús Maíz Apellániz ${ }^{5.8}$, \\ Emilio J. Alfaro ${ }^{5}$, Alfredo Sota ${ }^{5}$, Nolan R. Walborn ${ }^{6}$, and Christian Moni Bidin ${ }^{7}$ \\ ${ }^{1}$ Departamento de Física, Universidad de La Serena, Benavente 980, La Serena, Chile \\ 2 Instituto de Ciencias Astronómicas de la Tierra, y del Espacio (ICATE-CONICET), Av. España 1512 Sur, J5402DSP, San Juan, Argentina \\ ${ }^{3}$ Instituto de Astrofísica de La Plata, CONICET; Facultad de Cs Astronómicas y Geofísicas, Universidad Nac. de La Plata, \\ Paseo del Bosque S/N, La Plata. Argentina \\ ${ }^{4}$ Las Campanas Observatory. The Carnegie Observatories, Casilla 601. La Serena, Chile \\ ${ }^{5}$ Instituto de Astrofísica de Andalucía-CSIC, Glorieta de la Astronomía s/n, Granada 18008, Spain \\ ${ }^{6}$ Space Telescope Science Institute ${ }^{9}, 3700$ San Martin Drive, Baltimore, MD 21218 , USA \\ ${ }^{7}$ Departamento de Astronomía. Universidad de Concepción, Casilla 160-C, Concepción. Chile \\ Received 2009 August 28; accepted 2009 December 15; published 2010 January 20
}

\begin{abstract}
We present the analysis of high-resolution optical spectroscopic observations of the zero-age main-sequence $\mathrm{O}$ star Herschel 36 spanning six years. This star is definitely a multiple system, with at least three components detected in its spectrum. Based on our radial-velocity (RV) study, we propose a picture of a close massive binary and a more distant companion, most probably in wide orbit about each other. The orbital solution for the binary, whose components we identify as $\mathrm{O} 9 \mathrm{~V}$ and $\mathrm{B} 0.5 \mathrm{~V}$, is characterized by a period of $1.5415 \pm 0.0006$ days. With a spectral type $07.5 \mathrm{~V}$, the third body is the most luminous component of the system and also presents RV variations with a period close to 498 days. Some possible hypotheses to explain the variability are briefly addressed and further observations are suggested.
\end{abstract}

Key words: binaries: close - stars: early-type - stars: individual (Herschel 36)

Online-only material: machine-readable table

\section{INTRODUCTION}

The O-type star Herschel 36 (Her 36) is located in the M8 Nebula, a high-mass star-forming region at a distance of $1.3 \mathrm{kpc}$ (Arias et al. 2006). This O7.5 V(n) star (Walborn 1982 ) is responsible for the ionization of the optically brightest part of M8, known as the Hourglass Nebula. A very young infrared cluster is found around Her 36 (Arias et al. 2006). The ultracompact H II region G5.97-1.17, a candidate "proplyd" similar to those seen in the Orion Nebula (Stecklum et al. 1998), is located 2.7 distant from Her 36. Recently, high-resolution infrared imaging of the region also revealed the existence of a compact source at 0.'25 southeast of the star, which harbors an early B-type embedded star (Goto et al. 2006). Her 36 and its close neighbors are probably part of a Trapezium-like stellar system.

It seems clear that most, or even all, massive stars form in clusters and $\mathrm{OB}$ associations (de Wit et al. 2005). After a few hundred thousand years, they become optically visible. Only few, if any, of the massive stars are found as single objects and, on average, massive stars have more than one companion. Ignoring the multiplicity of massive stars can introduce serious problems when comparing observations and stellar evolution theory.

Although it is often stated that zero-age main-sequence (ZAMS) O stars should not be and are not observed optically, Her 36 appears as a possible bona fide ZAMS O star (Walborn 2007), based on the appearance of its optical spectrum, the association with a dense, dusty nebular knot, and its observed subluminosity (Arias et al. 2006). Because of all these aspects, Her 36 and the Hourglass have been many times compared to $\theta^{1}$

\footnotetext{
8 Ramón y Cajal Fellow.

9 STScI is operated by AURA. Inc.. under NASA contract NAS 5-26555.
}

Ori $\mathrm{C}$ and the Orion Trapezium. Her 36 has also been extensively studied because it shows an "anomalous" extinction curve with $R_{V}=5.36$ (Arias et al. 2006), which represents one of the highest $R_{V}$ values known (cf. Fitzpatrick \& Massa 2009).

A long-term spectroscopic monitoring of Galactic $\mathrm{O}$ and Wolf-Rayet of the Nitrogen sequence (WN) stars is being carried out (Gamen et al. 2008), with the main aim of establishing the multiplicity status of these objects. As part of this observational campaign, Her 36 has been systematically observed. Here, we present the results of the analysis of the data, which demonstrate conclusively that Her 36 is a complex multiple system, with outstanding characteristics.

\section{OBSERVATIONS AND DATA REDUCTION}

Table 1 presents the journal of the observations used in this work. Most of them came from the monitoring campaign of Galactic $\mathrm{O}$ and $\mathrm{WN}$ stars and were obtained between 2005 April and 2009 May at the observatories of Las Campanas (LCO) and La Silla, in Chile. A total of 33 high-resolution spectra were obtained at LCO; 32 of those were taken with the échelle spectrograph at the $2.5 \mathrm{~m}$ Du Pont Telescope and the other with the MIKE at the $6.5 \mathrm{~m}$ Magellan II (Clay) telescope. Twentysix more spectra were acquired with FEROS at ESO La Silla. Four additional spectra were taken at the Complejo Astronómico El Leoncito (CASLEO), Argentina, in observing runs prior to 2005, using the REOSC SEL Cassegrain spectrograph in cross-dispersion mode. Technical details of the observations and data reduction are presented by R. H. Barbá et al. (2010, in preparation)

\section{THE OPTICAL SPECTRUM}

Besides the Balmer series, the spectrum of Her 36 shows many absorption lines characteristic of hot massive stars, such 

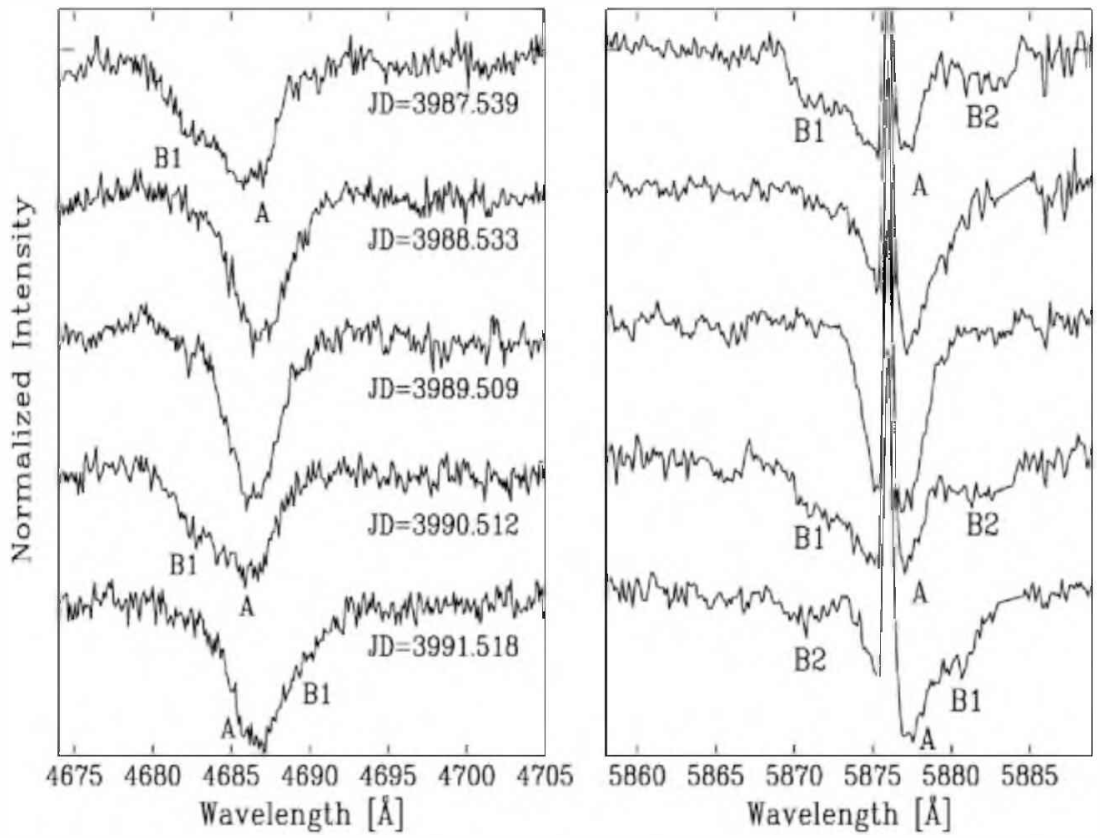

Figure 1. Variations in the He II $\lambda 4686$ (left panel) and He I $\lambda .5876$ (right panel) line profiles, observed in LCO/Du Pont spectrograms obtained in five consecutive nights. The spectra are labeled with the corresponding Julian Date (JD) in the format HJD-2,450,000. All the spectra are normalized to unity and have equal scales. The separation between continuum levels is $10 \%$ of the normalized flux. A, B1, and B2 refer to the components described in the text. The strong emission line superimposed on the He I absorption is associated with the Hourglass Nebula.

Table 1

Radial-velocity Measurements of the Stellar Components Observed in the Spectra of Her 36

\begin{tabular}{|c|c|c|c|c|c|c|c|c|}
\hline \multirow[t]{2}{*}{ No. } & \multirow{2}{*}{$\begin{array}{c}\text { HJD } \\
-2.450 .000\end{array}$} & \multicolumn{2}{|c|}{ Не II 4686} & \multicolumn{3}{|c|}{ He I 5876} & \multirow[t]{2}{*}{ Telescope } & \multirow[t]{2}{*}{ Instrumen } \\
\hline & & $\mathrm{A}$ & B1 & $\mathrm{A}$ & $\mathrm{B} 1$ & $\mathrm{~B} 2$ & & \\
\hline 1 & 2471.6791 & -10.2 & 126.0 & $\ldots$ & $\cdots$ & $\ldots$ & Casleo-2.15 & REOSC \\
\hline 2 & 2835.6685 & -58.8 & 92.9 & $\ldots$ & $\ldots$ & $\ldots$. & Casleo-2.15 & REOSC \\
\hline 3 & 2836.7348 & -72.5 & 218.4 & -65.0 & 256.8 & -229.5 & Casleo-2.15 & REOSC \\
\hline 4 & 2837.6846 & -54.5 & -146.9 & $\ldots$ & $\ldots$ & $\ldots$ & Casleo-2.15 & REOSC \\
\hline 5 & 3490.8852 & 26.4 & -128.7 & $\ldots$ & $\ldots$ & ... & LCO-DP & Echelle \\
\hline 6 & 3491.9145 & 13.6 & 205.3 & 16.6 & 213.8 & -336.1 & LCO-DP & Echelle \\
\hline 7 & 3491.9199 & 19.3 & 210.5 & 18.6 & 231.4 & -338.84 & LCO-DP & Echelle \\
\hline 8 & 3772.8789 & -47.0 & 50.3 & $\ldots$ & $\ldots$ & .. & LCO-DP & Echelle \\
\hline 9 & 3873.9131 & -50.9 & 91.2 & $\ldots$ & $\ldots$ & $\ldots$ & LCO-DP & Echelle \\
\hline 10 & 3874.9028 & -44.9 & -175.5 & -46.9 & -132.5 & 347.3 & LCO-DP & Echelle \\
\hline
\end{tabular}

Note. Rest wavelengths used in the determination of the RVs are $4685.65 \mathrm{~A}$ and $5875.65 \mathrm{~A}$, respectively.

(This table is available in its entirety in a machine-readable form in the online journal. A portion is shown here for guidance regarding its form and content.)

as those of He I, He II, C III and IV, O III and Si IV. Some diffuse interstellar bands at $4430 \mathrm{~A}, 4500 \mathrm{~A}, 5780 \mathrm{~A}$, and $5797 \AA$, as well as a number of interstellar absorption lines due to $\mathrm{Ca}$ II and $\mathrm{Na}$, are also evident. Narrow strong nebular emission lines, associated with the Hourglass Nebula, are observed, some of them superimposed on the Balmer and He I absorptions.

The spectrum of Her 36 was monitored during two to six consecutive nights in each of the observing runs. The first result that came from the inspection of our high-resolution data is that the spectrum of this star is highly variable.

The spectral lines of Her 36 show large variations in both line-profile shape and radial velocity (RV), on a timescale of hours. Even if all the absorption lines appear appreciably broadened, in good agreement with the qualifier (n) in the spectral classification by Walborn (1982), the profiles of the most prominent ones alternate between more or less normal and unusually broad. When observed with enough resolution, the lines are clearly not single: at different times and in different lines, one, two, or three components are detected, showing that this star is actually a multiple system.

We present the variations in the Hе II $\lambda 4686$ line in the left panel of Figure 1. This plot includes the data from five consecutive nights. The morphology observed varies from a very deep single profile to a well-defined double line. At first sight, however, the line-profile morphology does not seem to be strictly repeatable. Large RV variations accompany all these changes. We will refer to the main and secondary absorptions as "component $\mathrm{A}$ " and "component $\mathrm{B} 1$," respectively.

An even more complex behavior is observed in the He I absorption lines. The right panel of Figure 1 shows the spectral variations in the He I 25876 line. Now three absorption components (labeled as A, B1, and B2) can be clearly identified. Whereas components A and B1 correlate with those observed in the He II $\lambda 4686$ profile, the component B2 is undetected in the He II lines, indicating that the latter star is cooler than its companions. The RV of the main component A changes rather 
slowly, but the variations observed for components B1 and B2 are remarkably large. Note that the positions of the components B1 and B2 practically invert from the fourth to the fifth night.

Apart from the He II $\lambda 4686$ and He I $\lambda 5876$ lines, several other photospheric features, such as He I $\lambda 4471$, He II $\lambda 5411$, C III $\lambda 4650$, and C IV $\lambda 5812$, were also measured. We found correlated variations in profile shape and RV in most of these lines.

Finally, we considered the least blended spectra in order to obtain a spectral type for each of the three stellar components detected in the spectrum of Her 36. The spectral classification was performed by comparison of the spectrum with the OB star atlas of Walborn \& Fitzpatrick (1990). We adopt the spectral types $07.5 \mathrm{~V}, \mathrm{O} 9 \mathrm{~V}$, and B0.5 V for components A, B1, and B2, respectively.

\section{A PICTURE FOR THE MULTIPLE SYSTEM HER 36}

The fact that all measured photospheric lines show similar profile and RV variations suggests that the RV variations detected in the spectrum of Her 36 are actually caused by the orbital motion of, at least, three stellar components. We propose that the system is composed of a close O-type binary and a third more distant companion, also of spectral type $O$. The binary and the third body are very likely in wide orbit about each other.

\subsection{The Close Pair B1+B2}

The behavior observed in the He I $\lambda 5876$ line suggests that the components $\mathrm{B} 1$ and $\mathrm{B} 2$ are constituents of a double-lined spectroscopic binary. When we refer to this binary system, we will simply use the term "system B."

As mentioned before, the component B2 is not detected in He II. Moreover, only in the He I $\lambda .5876$ line, the profile features appear separated enough to obtain a reliable measurement of the RV of each component. Thus we considered only this line to determine the orbital solution for the stellar system B. The RV measurements used in the determination of the orbital solution are presented in Table 1 (Columns 6 and 7). We determined the RVs of the different components of the He I $\lambda 5876$ line by fitting them with adequate sums of Gaussian profiles. To do this, we considered four Gaussian functions: one for each of the stellar components A, B1, and B2, as well as an additional one to take into account the strong nebular emission. First, we used the least blended spectra to determine the intensities and widths of the three stellar blends and then repeated the disentangling by keeping these parameters fixed. Thus the deconvolution of Gaussian profiles yielded the central wavelengths of the four components (three stellar and one nebular) of the He I $\lambda 5876$ line as adjusted parameters. In spite of the complexity of this blend, the fits were highly satisfactory in most of the cases. Being conservative, we estimate that the measurement errors in the RVs of the stellar components are no larger than $10 \mathrm{~km} \mathrm{~s}^{-1}$.

To derive the period of the orbital motion, we applied a variety of methods to the RV measurements corresponding to the components B1 and B2. These routines include the Lafler \& Kinman (1965) method and subsequent modifications of it (Marraco \& Muzzio 1980, MM80), as well as information entropy minimization (Cincotta et al. 1995, CMN95). We considered three RV data sets: (1) the measurements of the primary component, B1, alone; (2) the measurements of the secondary component, B2, alone; and (3) the measurements of both components together. Based on the resulting periodograms, we adopted $P=1.54$ days as the best estimate for the period.
Table 2

Orbital and Physical Parameters of the System B

\begin{tabular}{lc}
\hline \hline \multicolumn{1}{c}{ Parameter } & Value \\
\hline$P_{S}$ (days) & $1.5415 \pm 0.0006$ \\
$T_{V \max }(\mathrm{HJD}-2,450,000)$ & $4248.8053 \pm 0.0061$ \\
$V_{o}\left(\mathrm{~km} \mathrm{~s}^{-1}\right)$ & $6.3 \pm 3.6$ \\
$K_{1}\left(\mathrm{~km} \mathrm{~s}^{-1}\right)$ & $201.4 \pm 5.8$ \\
$K_{2}\left(\mathrm{~km} \mathrm{~s}^{-1}\right)$ & $327.3 \pm 5.8$ \\
$a_{1} \operatorname{sin~} i_{B}(\mathrm{~km})$ & $(42.7 \pm 1.2) \times 10^{5}$ \\
$a_{2} \sin i_{B}(\mathrm{~km})$ & $(69.4 \pm 1.2) \times 10^{5}$ \\
$M_{1} \sin ^{3} i_{B}\left(M_{\odot}\right)$ & $14.6 \pm 1.3$ \\
$M_{2} \sin ^{3} i_{B}\left(M_{\odot}\right)$ & $9.0 \pm 1.3$ \\
$Q\left(M_{2} / M_{1}\right)$ & $0.61 \pm 0.03$ \\
$\operatorname{rms}\left(\mathrm{km} \mathrm{s}^{-1}\right)$ & 31.0 \\
\hline
\end{tabular}

Notes. Parameters derived from the RVs of the He I $\lambda .5876$ line and assuming a circular orbit. $T_{V \max }$ refers to the maximum velocity of the primary star B1.

To derive the orbital elements of the system $B$, we used a modified version of the code originally written by Bertiau \& Grobben (1969), adopting the value $P_{0}=1.54$ days as starting estimate of the period and considering the same data sets as previously described. After some trials with general eccentric fits, a circular orbit was assumed for the system. The best orbital solution is presented in Table 2 and illustrated by the RV curves in the upper panel of Figure 2.

\subsection{The Most Luminous Star A}

The star $\mathrm{A}$ is the most luminous component of the system and dominates the optical spectrum of Her 36. Although not so evident, it also presents RV variations. Careful inspection of the data suggests the presence of a long-term (a few hundred days) variability.

To study the spectral variations, we used the RV measurements of the He $I$ II $\lambda 4686$ line. This line appears as one of the most useful features for investigating possible periodicities because of being less contaminated by other features or the background nebular emission, and, mainly, because of showing only two stellar components: A and B1. As in the case of the He I $\lambda 5876$ line, we determined the RVs of the different components by performing multiple Gaussian fits. These measurements are presented in Columns 3 and 4 of Table 1. Due to the strong overlap of the line components, our initial disentangling attempts yielded systematic shifts of the central position of the component $A$, which at first we erroneously interpreted as short-term periodic RV variations. However, this effect was fully corrected when we repeated the disentangling by keeping the intensities and widths of the Gaussian profiles fixed, in order that the wavelength positions of A and B1 were the only parameters adjusted in the fit. As a result, we obtained very good quality fits and reliable RVs, with typical measurement errors no larger than $10 \mathrm{~km} \mathrm{~s}^{-1}$.

To estimate the possible period of the variability of $A$, we applied the same period-finding routines as in Section 4.1, i.e., the methods from MM80 and CMN95, to the corresponding RV measurements, and we also performed the Lomb-Scargle (LS) periodogram search (Scargle 1982). A number of possible values of the period can be identified from the periodograms peaks, the most relevant corresponding to $P=508 \pm 15$ days. An additional value of $P=206$ days is also strong but less significant, and cannot be discarded a priori.

Considering the values from the periodogram peaks as starting estimates of the period, we explored the possibility of 

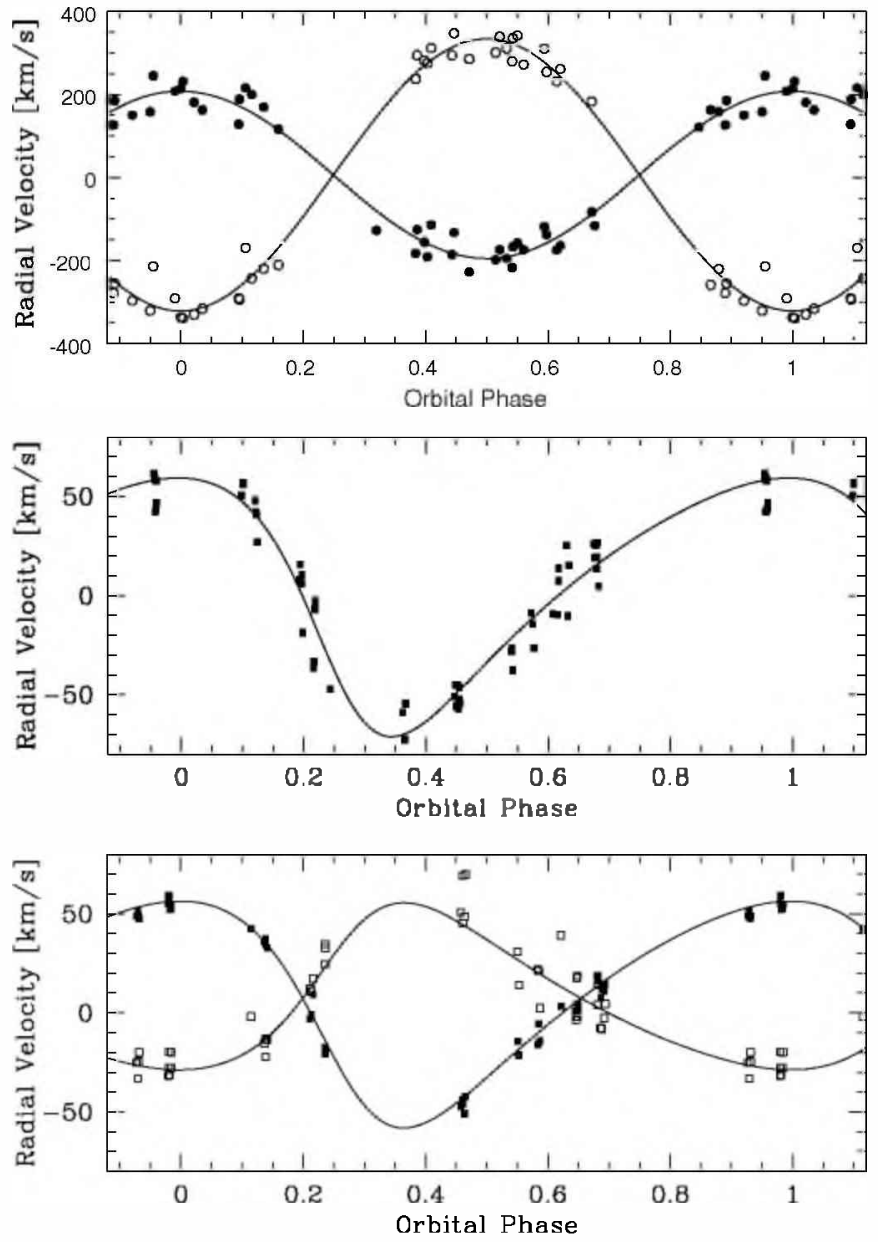

Figure 2. Upper panel: RV curves corresponding to the close stellar pair B1+B2. The filled and open circles indicate the observed RVs of the primary (B1) and secondary (B2), respectively. Zero phase is a time of maximum velocity for the primary B1. Middle panel: the RV curve derived from the measurements of the strongest component (component A) of the He in $\lambda .4686$ line. Lower panel: RV curves corresponding to a joint orbital solution obtained from the He I $\lambda 5876$ line, considering the RVs of the component $\mathrm{A}$ for the primary (filled squares) and the systemic velocities of the system B for the secondary (open squares). The orbital parameters that characterize these solutions are listed in Table 3. Zero phase is a time of maximum velocity for the component $\mathrm{A}$.

deriving an orbital solution for the motion of the component A. For this purpose, we applied the same code as in Section 4.1 to the RVs of the component A of the He II $\lambda 4686$ line. The resulting solution is presented in the second column of Table 3 and illustrated by the RV curve in the middle panel of Figure 2. An independent solution based on the RV measurements of the component A of the He I $\lambda 5876$ line (Column 5 of Table 1 ) was also calculated, and it is presented in the third column of Table 3. It can be verified that, within the errors, both solutions represent essentially the same orbit.

\section{SUMMARY AND DISCUSSION}

The main finding of this study is the multiple nature of the ZAMS object Her 36. The absorption lines in the spectrum of this star are clearly not single. We arrived at the conclusion that Her 36 presents at least three stellar components that we labeled as $\mathrm{A}, \mathrm{B} 1$, and $\mathrm{B} 2$, and classified as $07.5 \mathrm{~V}, 09 \mathrm{~V}$, and $\mathrm{B} 0.5 \mathrm{~V}$, respectively.

Arias et al. (2006) determined the distance and reddening to several early-type stars in the Hourglass region, including
Her 36. As mentioned in Section 1, Her 36 that was considered as a single $07.5 \mathrm{~V}$ star in their calculus fell below the calibration of its luminosity class. The here demonstrated multiplicity of Her 36 makes the stars that compose this triple system even much more subluminous individually. Thus, if we adopt the absolute magnitudes for ZAMS OB stars from Hanson et al. (1997), the combined $M_{V}$ value obtained is similar to that quoted by Arias et al. (2006). This fact adds additional support to the hypothesis that the stellar components of this system may be on the ZAMS

We measured the most prominent features in the spectrum of Her 36, finding correlated variations in profile shape and RV. Based on our RV study, we proposed that Her 36 is composed of a close massive binary, the stellar pair B1+B2 (system B), and a companion, the component $\mathrm{A}$, which is the most luminous star of the system and dominates the optical spectrum.

Considering the RV measurements of the He I $\lambda 5876$ line, we derived a set of orbital elements for the system B. The best solution is characterized by a circular orbit with a period $P_{S}=1.5415 \pm 0.0006$ days, and semi-amplitudes $K_{1}=201.4$ $\mathrm{km} \mathrm{s}^{-1}$ and $K_{2}=327.3 \mathrm{~km} \mathrm{~s}^{-1}$. We can use the obtained minimum masses $M \sin ^{3} i$ to estimate the inclination of the system $B$. The values of 14.6 and $9.0 M_{\circ}$, derived for the primary and the secondary, respectively, are compared with the typical masses of $\mathrm{O} 9 \mathrm{~V}$ and $\mathrm{B} 0.5 \mathrm{~V}$ stars. According to the values for detached eclipsing systems from Gies (2003) (19.0 $M_{\odot}$ for O9 $\mathrm{V}$ and $11.7 M_{\odot}$ for $\mathrm{B} 0.3 \mathrm{~V}$ ), the system must have a large inclination (larger than $67^{\circ}$ ), which means that it could possibly undergo eclipses. Unfortunately, no photometric study of Her 36 exists at present. Future photometric monitoring will certainly be valuable.

The component A also shows RV variations. Independent RV measurements of the He II $\lambda 4686$ and the He I $\lambda .5876$ lines yield essentially the same relatively eccentric $(e=0.3$ ) orbit, characterized by a period close to 498 days and a semi-amplitude of the order of $65 \mathrm{~km} \mathrm{~s}^{-1}$

We feel that the component $\mathrm{A}$ and the close binary system $B$ are in wide orbit about each other. The long-term periodicity observed in the RVs of the component A must then be related to the mutual orbit of both objects. If that is the case, and we are indeed in the presence of a triple (hierarchy $2+1$ ) system, then we should expect the systemic velocity of the binary B to change with a period $P_{L} \approx 498$ days. To prove this hypothesis, we computed a joint orbital solution considering the RVs of the component A of the He I $\lambda 5876$ line for the primary, and the systemic velocities of the system $\mathrm{B}, v_{B}$, for the secondary. The $v_{B}$ values were calculated according to the basic expression $v_{s}=\left(M_{1} v_{1}+M_{2} v_{2}\right) /\left(M_{1}+M_{2}\right)$, where $M_{1}$ and $M_{2}$ are the minimum masses from Table 2 , and $v_{1}$ and $v_{2}$ are the RVs of the components B1 and B2 of the $\mathrm{He}$ I $\lambda 5876$ line, respectively. The resulting solution is characterized by the parameters in the last column of Table 3 and the RV curves in the lower panel of Figure 2. Although with somewhat larger dispersion, these curves represent practically the same orbit as those derived from the RV data of A alone, as can be easily verified by confronting the orbital parameters listed in the different columns of Table 3. We note here that the joint solution was obtained in a completely independent manner, supporting the idea of the mutual orbital motion between $\mathrm{A}$ and $\mathrm{B}$.

Comparing the projected mass of the system B from Table 3 with the projected total mass of the close pair $\mathrm{B} 1+\mathrm{B} 2$ from Table 2 , it can be shown that $\sin i_{A}=1.03 \sin i_{B}$. This means that the inclinations of the two orbital planes satisfy either 
Table 3

Orbital and Physical Parameters of the Multiple System Her 36

\begin{tabular}{|c|c|c|c|}
\hline Parameter & $\begin{array}{c}\text { He II } \lambda 4686 \\
\mathrm{~A}\end{array}$ & $\begin{array}{c}\text { He I } \lambda 5876 \\
\mathrm{~A}\end{array}$ & $\begin{array}{c}\text { He I } \lambda 5876 \\
A+B\end{array}$ \\
\hline$P_{L}$ (days) & $498.3 \pm 3.7$ & $496.3 \pm 2.2$ & $493.3 \pm 3.2$ \\
\hline$e$ & $0.29 \pm 0.04$ & $0.30 \pm 0.03$ & $0.25 \pm 0.03$ \\
\hline$\omega(\operatorname{deg})$ & $126.0 \pm 6.3$ & $126.9 \pm 3.4$ & $124.5 \pm 5.4$ \\
\hline$T_{o}[\mathrm{HJD}-2,450,000]$ & $4279.5 \pm 7.5$ & $4278.6 \pm 3.9$ & $4275.6 \pm 6.5$ \\
\hline$T_{V \max }[\mathrm{HJD}-2,450,000]$ & $4147.5 \pm 7.5$ & $4146.7 \pm 3.9$ & $4141.5 \pm 6.5$ \\
\hline$V_{o}\left(\mathrm{~km} \mathrm{~s}^{-1}\right)$ & $5.3 \pm 1.8$ & $4.4 \pm 0.9$ & $7.4 \pm 0.8$ \\
\hline$K_{A}\left(\mathrm{~km} \mathrm{~s}^{-1}\right)$ & $65.2 \pm 3.1$ & $61.2 \pm 2.2$ & $57.2 \pm 2.4$ \\
\hline$K_{B}\left(\mathrm{~km} \mathrm{~s}^{-1}\right)$ & $\cdots$ & $\ldots$ & $42.1 \pm 2.4$ \\
\hline$a_{A} \sin i_{A}(\mathrm{~km})$ & $(42.7 \pm 3.0) \times 10^{7}$ & $(39.8 \pm 1.9) \times 10^{7}$ & $(37.5 \pm 2.1) \times 10^{7}$ \\
\hline$a_{B} \sin i_{A}(\mathrm{~km})$ & $\ldots$ & $\ldots$ & $(27.6 \pm 2.0) \times 10^{7}$ \\
\hline$f(M)\left(M_{\odot}\right)$ & $12.5 \pm 2.4$ & $10.22 \pm 1.5$ & $\ldots$ \\
\hline$M_{A} \sin ^{3} i_{A}\left(M_{\circlearrowleft}\right)$ & $\ldots$ & $\ldots$ & $19.2 \pm 6.1$ \\
\hline$M_{B} \sin ^{3} i_{A}\left(M_{\odot}\right)$ & $\cdots$ & $\ldots$ & $26.0 \pm 6.3$ \\
\hline$Q\left(M_{B} / M_{A}\right)$ & $\cdots$ & $\cdots$ & $1.36 \pm 0.13$ \\
\hline $\mathrm{rms}\left(\mathrm{km} \mathrm{s}^{-1}\right)$ & 10.3 & 3.6 & 7.0 \\
\hline
\end{tabular}

Notes. Columns 2 and 3 present the orbital parameters derived from the RVs of the component $A$ alone, considering the He II $\lambda 4686$ and the He I $\lambda 5876$ lines, respectively. Column 4 presents a joint orbital solution obtained from the He I $\lambda 5876$ line, considering the RVs of the component A for the primary and the systemic velocities of the system B for the secondary.

$i_{B} \simeq i_{A}$ or $i_{B} \simeq 180^{\circ}-i_{A}$. In the first case, the inner and the outer orbits of the triple system will be nearly coplanar, whereas in the last case the relative inclination between the orbital planes, $\phi$, will lie between $180^{\circ}-2 i_{A} \leqslant \phi \leqslant 180^{\circ}$ (Fekel 1981). The relative orientation of the orbits in a multiple system is of particular interest because of its direct relationship with the conditions at the epoch of formation. Thus, the study of a triple system as young as Her 36 can provide important clues to the formation processes of massive stellar systems.

On the other hand, comparing the minimum mass for the primary A $\left(19.2 M_{\circ}\right)$ to typical masses of $07.5 \mathrm{~V}$ stars from Gies (2003), we estimate that $i_{A}$ is most probably larger than $70^{\circ}$. The component $\mathrm{A}$ has the largest individual mass of the three stars. Its mass is however fairly comparable with the total mass of the inner binary B. According to Table 3, the minimum mass of the triple system is $19.2+26.0=45.2 M_{\odot}$. Assuming a minimum inclination $i_{A} \simeq 70^{\circ}$, we conclude that the total mass of Her 36 is probably no larger than $54 M_{\circ}$.

The triple ZAMS system Her 36 is really peculiar, and it undoubtedly deserves additional study. Clearly, further observations are required before a complete characterization of the orbits can be given and a definitive model for the system can be derived. Spectroscopic observations with higher signal-tonoise ratio, as well as an exhaustive program of photometric monitoring, seem essential.

We would like to thank our anonymous referee whose critical remarks helped us to substantially improve this Letter. J.I.A. and R.H.B. acknowledge financial support from DIULS through projects No. PI07101 and CD08102, respectively and J.I.A. from Fondo ALMA para el Desarrollo de la Astronomía CONICYT No. 31050004. J.M.A., E.A., and A.S. acknowl- edge support from the Spanish Government MICINN through grant AYA2007-64052 and from Consejería de Educación y Ciencia (Junta de Andalucía) through TIC-101 and TIC-4075. J.M.A. acknowledges support from the Ramón y Cajal Fellowship program and FEDER funds. C.M.B. acknowledges the Chilean Centro de Excelencia en Astrofísica y Tecnologías Afines (CATA). This work relies on data taken at the La Silla Observatory under Program IDs 077.B-0348(A), 079.D0564(A), 079.D-0564(C), 081.D-2008(A), 081.D-2008(B), and 083.D-0589(A).

\section{REFERENCES}

Arias, J. I., Barbá, R. H., Maíz Apellániz, J., Morrell, N. I., \& Rubio, M. 2006. MNRAS, 366, 739

Bertiau, F., \& Grobben, J. 1969, Ric. Astron. Sp. Vaticana, 8. 1

Cincotta, P. M., Méndez, M., \& Nuñez, J. A. 1995, ApJ, 449, 231

de Wit. W. J., Testi, L., Palla, F.. \& Zinnecker, H. 2005, A\&A. 437, 247

Fekel. F. C. 1981. ApJ. 246. 879

Fitzpatrick. E. L.. \& Massa, D. 2009. ApJ, 699. 1209

Gamen, R. C., Barbá, R. H., Morrell, N. I., Arias, J. I., \& Maíz Apellániz, J. 2008, RevMexAAC, 33, 54

Gies, D. R. 2003, in IAU Symp 212, A Massive Star Odyssey: From Main Sequence to Supernova, ed. K. van der Hucht. A. Herrero. \& C. Esteban (Dordrecht: Kluwer), 91

Goto, M., Stecklum, B., Linz, H., Feldt, M., Henning, Th., Pascucci, I., \& Usuda, T. 2006, ApJ, 649, 299

Hanson, M. M., Howarth. I. D., \& Conti, P. S. 1997, ApJ, 489, 698

Lafler. J., \& Kinman. T. D. 1965. ApJS. 11. 216

Marraco, H. G., \& Muzzio, J. C. 1980, PASP, 92, 700

Scargle, J. D. 1982, ApJ, 263, 835

Stecklum, B.. et al. 1998. AJ, 115, 767

Walborn. N. R. 1982, AJ, 87, 1300

Walborn. N. R., \& Fitzpatrick. E. L. 1990. PASP. 102, 379

Walborn, N. R. 2007, in Proc. STScI Symp., 20 Massive Stars: From Pop III and GRB to the Milky Way, ed. M. Livio \& E. Villaver (Cambridge: Cambridge Univ. Press), 167 